\title{
Accuracy of the French Administrative Database to Describe Patients' Medication and Primary Care Visits: A Validation Study
}

\author{
Anaïs PAYEN ${ }^{\mathrm{a}, 1}$, Claire GODARD-SEBILLOTTE ${ }^{\mathrm{b}}$, Julien SOULA ${ }^{\mathrm{a}}$, David \\ VERLOOP $^{c}$, Marie-Marguerite DEFEBVRE ${ }^{\mathrm{c}}$, \\ Delphine DAMBRE ${ }^{\mathrm{d}}$ and Jean-Baptiste BEUSCART ${ }^{\mathrm{a}}$ \\ a Univ. Lille, CHU Lille, ULR 2694 - METRICS : Évaluation des technologies de \\ santé et des pratiques médicales, F-59000 Lille, France \\ ${ }^{\mathrm{b}}$ Department of Family Medicine, McGill University, Montreal, Canada \\ ' Agence Régionale de Santé Hauts-de-France, Lille, France \\ ${ }^{\mathrm{d}}$ Service médecine polyvalente, Centre Hospitalier de Saint-Amand-les-Eaux, France
}

\begin{abstract}
Objective: To evaluate the accuracy of the French health administrative database to describe patients' medication and primary care visits, in the context of a transitional care intervention including an in-hospital medication reconciliation followed by a structured community follow-up by the patient's general practitioner and pharmacist. Design: A retrospective cohort study of older persons enrolled in the transitional care intervention between January 1st, 2015 and December 31st, 2018. Results: Only $46.1 \%$ of the community follow-up were timely billed, in the 3 months after the patient discharge. The sensitivity of the health administrative database to identify medications was $90.0 \%$. Its positive predictive value was $50.1 \%$. Conclusion: This study reveals that the French health administrative database was poorly reliable to identify both community follow-up and chronic medications.
\end{abstract}

Keywords. Health administrative database, older persons, medication reconciliation, claims data, transitional care.

\section{Introduction}

In many countries, health administrative databases have been used to conduct surveillance and research activities: i.e. describing diseases' prevalence, and incidence, or patients' trajectories [1]. Their accuracy to describe these indicators varies widely depending on the type of data, their source and use [2]. The accuracy of the health administrative database to describe patients' medication and primary care visits, in the context of a transitional care intervention, has never been assessed. The PAERPA (Personnes âgées En Risque de Perte d'Autonomie) intervention was an ideal setting to perform such an evaluation. This intervention was an innovative integrated care program

\footnotetext{
${ }^{1}$ Corresponding Author, Anaïs PAYEN: E-mail: anais.payen.etu@univ-lille.fr
} 
including actions at the macro-, meso- and micro-levels [3]. The PAERPA intervention included a transitional care intervention involving an in-hospital medication reconciliation followed by a structured community follow-up by the patient's general practitioner (GP) and pharmacist [4]. Medications and clinicians' visits were recorded in the intervention implementation file as well as in the health administrative database, allowing for the evaluation of the administrative database accuracy. The objective of this study was to evaluate the accuracy of the health administrative database to describe patients' medications and primary care visits, in the context of a transitional care intervention.

\section{Materials and Methods}

\subsection{Study design and ethics approvals}

We analyzed a retrospective cohort of older persons enrolled in the PAERPA intervention between January 1st, 2015 and December 31st, 2018 in the ValenciennoisQuercitain area in France. Informed consent was obtained from every participant: patients and health- care professionals. In line with the ethic approval granted by the French government, the data were accessed through the Hauts-de-France Regional Health Authority after registration with the French National Data Protection Commission (Commission Nationale de l'Informatique et des Libertés).

\subsection{The transitional care intervention}

The transitional care intervention included an in-hospital medication reconciliation followed by a structured community follow-up by the patient's GP and pharmacist in the 30-days after discharge. The medication reconciliation aimed at identifying chronic medication in the 90 days before hospital admission. Medications which were likely not active at admission (i.e. short courses of antibiotics) were not recorded in the medication reconciliation list [5]. The GP and pharmacist visits had to be billed within 3 months of patient's discharge. Each patient could be enrolled in the transitional care intervention once a year.

\subsection{Data sources}

\section{Intervention implementation file}

In the intervention implementation file were recorded socio-demographic, and clinical characteristics, as well as the community follow-up of every patient enrolled in the intervention and discharged alive. We extracted: year of birth, sex, date of hospital admission and discharge, medication reconciliation list, as well as community followup.

\section{Health administrative database}

The health administrative database is a linkage of health insurance database (SNIIRAM database), hospital database (PMSI database) and medical causes of death (CépiDC database) [6]. It records every drug delivered in the community, as well as primary care physicians and pharmacist visits. The data extracted were put into the Observational medical outcomes partnership (OMOP) format [7]. We extracted, for every person 
having at least one hospital stay in the study hospital during the study period, year of birth, sex, date of hospital admission and discharge, the list of drugs delivered within the 90 days prior to hospital admission, visits billed related to the intervention by GP and pharmacists until the January $1 \mathrm{st}, 2020$. GP and pharmacists were asked to bill their visits within the 3 months of patient hospital discharge. Billing had to be performed through a dedicated process different from their usual billing procedure.

\subsection{Cohort construction}

Under French regulations, person's unique identifying number, as well as name and date of birth were not accessible in the administrative database. Persons included in the transitional care intervention were matched probabilistically to persons identified in the health administrative database. Probabilistic matching is the recommended procedure to per- form analysis in the health administrative database and was based on year of birth, sex and dates of hospital admission and discharge [8].

\subsection{Analysis}

We described the time to billing by GP and pharmacists in the health administrative database. Indeed, according to the intervention implementation file every person received the complete community follow-up. Sensitivity and Predictive Positive Value could not be computed (false positives and true negatives equal to 0 ). Then we compared medications identified in the medication reconciliation list and the health administrative database. The medication reconciliation was considered to be the gold-standard [5]. Sensitivity and Predictive Positive Value were computed. It was not possible to determine the true negatives as they would correspond to all drugs that could be prescribed in France.

\section{Results}

Between 1st January 2015 and 31st December 2018, 328 transitional care interventions were performed at the study hospital according to the intervention implementation file. They were probabilistically matched with 291 (88.7\%) hospital stays identified in the health administrative database. Thirty-seven hospital stays were excluded because of i) absence of medication delivery recorded in the 90 days prior to hospital admission in the health administrative database or ii) lack of consistent criteria between the intervention implementation file and the health administrative database to perform the matching.

The 273 persons enrolled in these 291 interventions had a median age of $83.1 \pm 4.6$ years, $65.3 \%$ were women, and the median length of hospital stay was 9 days [6]. All of the 291 interventions had a completed community follow-up, according to the intervention implementation file. Only 134 (46.0\%) were timely billed within 3 months after hospital discharge, 50 (17.2\%) had no billing between 2015 and 2020 (Figure 1). The median billing time after hospital discharge was 2.4 months $[1,3,6]$. 


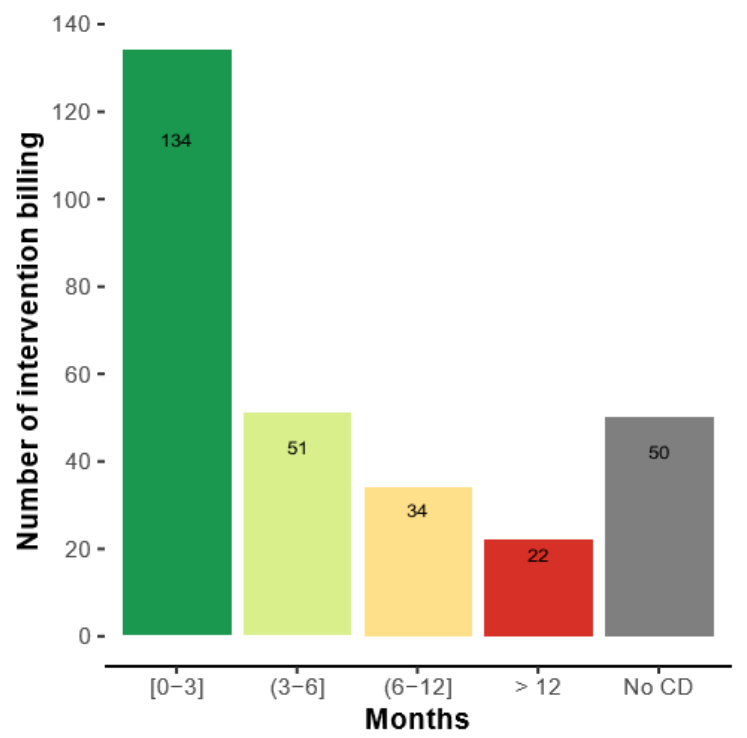

Figure 1. Duration in months between hospital discharge and billing by general practitioners and pharmacists for the transitional care intervention. (BD: Billing Data)

The sensitivity of the health administrative database to identify medications delivered in the 90 days prior to hospital admission was $90.0 \%$, i.e. $90.0 \%$ of unique ATC codes present in the intervention implementation file were found in the health administrative database. The positive predictive value was $50.1 \%$, one out of two ATC codes identified in the health administrative database was actually present in the medication reconciliations (Table 1).

Table 1. Number of ATC codes identified in the medication reconciliation extracted from the intervention implementation file, and in the health administrative database. (NA: Not Available)

\begin{tabular}{|l|l|l|l|}
\cline { 3 - 4 } \multicolumn{2}{c|}{} & \multicolumn{2}{|l|}{ Intervention implementation file } \\
\cline { 3 - 4 } \multicolumn{2}{|c|}{ Health administrative database } & $\begin{array}{l}\text { Number of codes } \\
\text { identified }\end{array}$ & $\begin{array}{l}\text { Number of codes } \\
\text { non-identified }\end{array}$ \\
\cline { 2 - 4 } & $\begin{array}{l}\text { Number of codes } \\
\text { identified }\end{array}$ & 2000 & 1994 \\
\cline { 2 - 4 } & $\begin{array}{l}\text { Number of codes } \\
\text { non-identified }\end{array}$ & 221 & NA \\
\hline
\end{tabular}

\section{Discussion}

This study reveals that the data from the French health administrative database were poorly reliable to identify both the community follow-up of the transitional care intervention and chronic medications identified through an in-hospital medication reconciliation. This is in contrast with the known accuracy of health administrative databases found in other countries with universal public health insurance. Less than half of the community follow-up were timely billed, with close to $20 \%$ which were never been billed. Using the health administrative database to assess the implementation of this 
transitional care intervention is not to be recommended. One of the possible explanations of the lack of billing or timely billing by community clinicians could be the complicated process to be followed to bill each visit (outside of their usual billing scheme), making it non-financially appealing to clinicians. The health administrative database had a high sensitivity, but a poor predictive positive value to identify chronic medications. Several hypotheses could explain these results. First, few treatments were not identified in the administrative database, meaning that they were not delivered to the person in the 90 days prior to hospital admission. Patients might be prescribed treatments, that they do not get from the pharmacy, either because they do not take them, or because they have already them at home. In France, pharmacists are forbidden to unpack drugs, leading some time to huge accumulations of drugs at patients' home [9]. The examination of the ATC codes that were not identified in the administrative database favors the second hypothesis (data not shown). Second, some treatment delivered and identified in the administrative database were not identified in the in-patient medication reconciliation. This may be explained by the fact that medication reconciliation only considered chronic and active treatments whereas in the health administrative database, all drugs appeared including short-term (e.g. antibiotics). We did not perform a sensitivity analysis considering only chronic treatment in the health administrative database, as many ATC codes can be both used as chronic or short-term treatment, for example corticosteroids in COPD. In order to improve the predictive positive value, it would be interesting to develop an algorithm to determine chronic treatments from the administrative health database. This concerns in particular medications that are dispensed at least 3 times a year, or medications that have a 3-month conditioning period. In conclusion, our study revealed that the health administrative database should be cautiously used to measure the implementation of a transitional care intervention and to identify medications in older people.

\section{References}

[1] Ficheur G, et al. Elderly Surgical Patients: Automated Computation of Healthcare Quality Indicators by Data Reuse of HER. Studies in Health Technology and Informatics. 2016;221:92-96.

[2] Koram N, et al. Validation Studies of Claims Data in the Asia-Pacific Region: A Comprehensive Review. Pharmacoepidemiology and Drug Safety. 2019;28(2):156-170.

[3] Briggs M, et al. Elements of Integrated Care Approaches for Older People: A Review of Reviews. BMJ Open 2018;8(4).

[4] Le Berre M, et al. Impact of Transitional Care Services for Chronically Ill Older Patients: A Systematic Evidence Review. Journal of the American Geriatrics Society. 2017;65(7): 1597-1608.

[5] Murphy EM, et al. Medication Reconciliation at an Academic Medical Center: Implementation of a Comprehensive Program from Admission to Discharge. American Journal of Health-System Pharmacy 2009;66.23: 2126-2131.

[6] Tuppin P. et al. Value of a National Administrative Database to Guide Public Decisions: From the SNIIRAM to the SNDS in France. Revue d'Épidémiologie et de Santé Publique 2017;65: 149-S167.

[7] Lamer A, et al. Transforming French Electronic Health Records into the Observational Medical Out- come Partnership's Common Data Model: A Feasibility Study. Applied Clinical Informatics. 2020;11(01):1322.

[8] Belhassen M, et al. 10-Year Comparative Follow up of Familial versus Multifactorial Chylomicronemia Syndromes. The Journal of Clinical Endocrinology \& Metabolism. 2020.

[9] Beuscart J-B, et al. Polypharmacy in Older Patients: Identifying the Need for Support by a Community Pharmacist. BMC Geriatrics 2019; 19(1). 